\title{
Peripheral biomarkers in bipolar disorder: a population-based study in young adults (Abstract)
}

Biomarcadores periféricos no transtorno bipolar: um estudo de base populacional em adultos jovens (Resumo)

Pedro Vieira da Silva

Programa de Pós-Graduação em Ciências Médicas: Psiquiatria na Universidade Federal do Rio Grande do Sul, 2011. Orientador: Flávio Kapczinski.

Correspondence: Pedro Vieira da Silva; Laboratório de Psiquiatria Molecular; Hospital de Clínicas de Porto Alegre; Rua Ramiro Barcelos 2350; $90035-903$ Porto Alegre RS - Brasil; E-mail:pedromaga@yahoo.com

\section{ABSTRACT}

Objective: The aim of this study was to confirm, in a sample of young adults from the general population, recent findings regarding the pathophysiology of bipolar disorder. The focus of this investigation was finding group differences in one neurotrophin, two markers of oxidative damage, two proinflammatory cytokines and one anti-inflammatory cytokine in participants with bipolar disorder, major depression and people without any mood episodes. Markers assessed here were brain-derived neurotrophic factor (BDNF), thiobarbituric acid reactive substances (TBARS), protein carbonyl content (PCC), tumor necrosis factor-alpha (TNF- $\alpha$ ), interleukin-6 (IL-6) and interleukin-10 (IL-10). Method: Individuals from the general population, previously included in a cross-sectional study ( $n=1560)$, with a positive screen for bipolar disorder were recruited, as well as two groups of controls. One had only depressive episodes and the other had no history of mood episodes. This yielded a sample of 231 participants that further underwent diagnostic confirmation with the Structured Clinical Interview for DSM-IV (SCID). All analyses included a check for bivariate associations, as well as an a priori multivariate model with sex, social class, current mood state, use of substances and SCID diagnoses as predictors. Results: The final sample included 55 participants with bipolar disorder, 82 with major depression and 94 healthy controls. Only a minority was using any psychiatric medications (9.6\%). Bipolar disorder was associated with higher PCC and TNF- $\alpha$ levels when compared to the control group. Major depression was also associated with higher PCC levels when compared to the control condition. Use of psychiatric medication was associated with lower TNF- $\alpha$ levels. Correlations between the same markers were not as strong as in clinical samples. Conclusions: Two broad conclusions are called for from these results. The first is that early-stage bipolar disorder is already associated with a pro-oxidant, proinflammatory state. The second is that these changes appear more subtle than those observed in typical late-stage, chronic patients, supporting the notion that a form of illness progression takes place. The main caveat is that these data are cross-sectional, not longitudinal, which precludes causal inferences as factors other than the bipolar illness can conceivably induce systemic toxicity.

Key words: bipolar disorder, mood disorders, pathophysiology, neurotrophins, inflammation markers, oxidative stress, general population, case-control.

\section{Severity and functional ability scale for amyotrophic lateral sclerosis patients (Abstract)}

Escala de gravidade e habilidade funcional em pacientes com esclerose lateral amiotrófica. (Resumo)

Marco Antonio Orsini

Departamento de Neurologia, Universidade Federal Fluminense (UFF), Rio de Janeiro RJ, Brazil, 2011

Orientador: Prof. Dr. Marcos Raimundo Gomes de Freitas.

Co-orientador: Prof. Osvaldo JM Nascimento.

Correspondence: Marco Antonio Orsini; Herotides de Oliveira 2/801; 24230-230 Niterói RJ - Brasil; E-mail: orsinimarco@hotmail.com

\section{ABSTRACT}

Introduction: Amyotrophic lateral sclerosis (ALS) is a progressive, degenerative disease that leads to the motor neurons depletion in the spinal cord anterior horn and pyramidal tract. Several evaluations have been proposed in order to provide a better follow-up and management of secondary complications. However, the biggest difficulty is to select a single instrument to objectively assess the neurological deficit, the 
functional independence level and, above all, one which will include such subjects in specific severity stages. Objective: To present a new tool for clinical and functional evaluation for ALS patients, the Severity and functional ability scale (SFAS), and discuss the clinical and functional profile of 98 patients between March 2007 and December 2009 at Antonio Pedro University Hospital (UFF) and the Deolindo Couto Institute of Neurology (UFRJ). Method: We used the SFAS as clinical and functional indicator. The modified El Escorial criteria were used to establish the diagnosis. The participants underwent a number of five quarterly assessments during the study period of one year. Results and discussion: The average age of the study group was 52.67 years \pm 12.043 years. The average time between the onset of first symptoms and seeking care services was $11.57 \pm 12.391$ months. The average time between first symptoms and the diagnosis was $20.54 \pm 8.413$ months. Of the 98 subjects tested, only 24 have completed all phases of the study. Muscle weakness was identified as the initial symptom in most cases. The patients showed progressive impairment of muscle strength, speech, swallowing, breathing and the severity stages. The disease was marked by different forms of initial presentation (speech, limbs, breathing, swallowing), progression speed and diverse clinical characteristics in our population. Conclusion: Knowledge of the clinical and functional ALS peculiarities, obtained by applying the SFAS, serves as a guide for proper monitoring of patients during the development stages and encourages the formulation of a theoreticalconceptual framework, aiming at solving problems in clinical practice.

Key words: amyotrophic lateral sclerosis, motor neuron, epidemiology. 